\title{
Hemolytic Anemia Due to Anti-A in Concentrated Antihemophilic Factor Preparations
}

\author{
L. A. Rosati, B. Barnes, H. A. Oberman, and J. A. Penner \\ From the Departments of Pathology and Internal Medicine (Simpson Memorial Institute), \\ University of Michigan, Ann Arbor, Michigan
}

Progreasive anemia developed in a type $A_{1}$ hemophiliac after he had received a large amount of commercial Factor VIII concentrate. Anti-A was found in the patient's serum as well as in eluates from his erythrocytes. Moreover, the product contained potent anti-A. A positive direct antiglobulin test and increased osmotic fragility with microspherocytosis were additional laboratory findings which suggested passive transfer of anti-A as the cause of the anemia. The possibility of anemia due to transfused anti-A or anti-B should be considered in patients, other than those of type $O$, who manifest progressive ancmia following treatment with large volumes of a pooled plasma product.

There have been several reports of progressive anemia due to $A B O$ antibodies infused with large volumes of pooled plasma as used for patients with extensive burns or hemophilia.1, 5,7 In a previous report we studied the lyophilized pooled plasma received by two hemophiliacs and noted the disparity between the potency of these antibodies and their serologic activity. ${ }^{4}$ Therapeutic implications of future transfusions for such patients were emphasized.

Recently we encountered a type A hemophiliac who became progressively anemic in the absence of overt blood loss, and whose blood was serologically incompatible with all tested type A donor units. This patient had not received pooled plasma, but had been transfused with large amounts of a high-potency concentrated Factor VIII. We are not aware of prior reports of erythrocyte sensitization and anemia due to such a product, although there has been documentation of hemolysis due to $\mathrm{ABO}$

Received for publication January 2, 1970; accepted February 3, 1970. antibodies in hemophiliacs treated with commercal Cohn Fraction I. ${ }^{2}$

\section{Case Repurt}

A 22-year-old man was admitted for evaluation and treatment of a pseudotumor of his right thigh. The diagnosis of hemophilia A was first established when he was eight years old, at which time unusual bleeding followed circumcision. During subsequent years he had bleeding complications. Frequently, some of these complications involved his right knee, and resulted in joint deformity and fracture. Although each hemorrhagic complication responded to therapy, a large pseudotomor developed subsequently and this was excised ten months before the present admission.

His most recent admission was prompted by an improperly fitting cast on the right leg. Admission hemoglobin was $15.8 \mathrm{~g} / 100 \mathrm{ml}$ and hematocrit was 44.5 per cent. He was type $A_{1}, \mathbf{R}$ h-positive. Roentgenograms revealed displacement of his right femur with respect to its articulation with the tibia. Subsequently, the femur was refractured and a closed reduction was carried out on the 26th hospital day. Treatment with a high-potency commercial Factor VIII concentrate* was instituted at the time of admission, and he had received 20,000 units by the day of operation. Two units of serologicallycompatible type $A$ whole blood were reserved for surgery, and no atypical antibodies were observed in the patient's serum. Blood was not required during this operation.

Unfortunately, a pseudotumor recurred in the area of the fracture. Excision was not possible and an above-knee amputation was performed on the 40th hospital day. At that time his hemoglobin was $14.5 \mathrm{~g} / 100 \mathrm{ml}$, hematocrit was 43 per cent, and he had received 43,000 units of the commercial Factor VIII concentrate.

Two serologically-compatible units of type A whole blood were given during the amputation. Atypical antibodies were not demonstrable at this time and, postoperatively, the patient continued to receive the commercial Factor VIII, 2,000 to 7,000 units per day.

The patient's hematocrit on the first and second

- Hemofil, Hyland Laboratories, Los Angeles, California. 
days after the amputation were 38 per cent and 33 per cent, respectively, but there was no evidence of overt blood loss until the fourth postoperative day when bleeding gradually developed at the amputation site. Three units of serologically-com. patible type $A$ whole blood were transfused without incident and no subsequent bleeding was detected. The hematocrit, however, continued to fall; by the ninth day after amputation it was 20 per cent.

The patient's erythrocytes continued to be type $A_{1}$, Rh-positive, but his serum now strongly agglutinated all type $\mathbf{A}$ cells, direct antiglobulin test was strongly positive, and anti-A was demonstrated in heat eluates. He had received 57,000 units of the Factor VIII preparation by the ninth day after the amputation in addition to the 43,000 units given preoperatively. Additional laboratory findings included: total serum bilirubin, $1.0 \mathrm{mg} / 100 \mathrm{ml}$; serum hemoglobin, $7.8 \mathrm{mg} / 100 \mathrm{ml}$; haptoglobin binding capacity, less than $20 \mathrm{mg} / 100 \mathrm{ml}$; reticulocyte count, 18.2 per cent. Microspherocytes were observed, accompanied by increased osmotic fragility. The latter was especially clear after incubation for 24 hours at $37 \mathrm{C}$ when initial hemolysis was noted in 0.80 per cent sodium chloride and complete hemolysis was found in 0.40 per cent sodium chloride.

Two units of serologically-compatible type $O$, Rh-positive packed red blood cells were transfused without complication, and during the remainder of his hospitalization he received Factor VIII cryoprecipitates prepared from type AB donors. No other blood products were administered. One week after the transfusion of the type $O$ packed cells his hemoglobin was $8.8 \mathrm{~g} / 100 \mathrm{ml}$ and his hematocrit was 29.5 per cent. He continued to improve, the positive antiglobulin test became progressively weaker during the remainder of his hospital stay, and he was discharged on the 85th hospital day.

Not all lots of the commercial Factor VIII prepa. ration received by this patient were available for testing purposes, but tests for anti-A were performed on two which represented much of what was received by the patient. The strength of anti-A was determined by indirect antiglobulin titer following partial neutralization with group-specific substance. ${ }^{6}$ The saline-agglutinating anti-A activity of both these lots was consistently below $1: 64$, but the titer value by indirect antiglobulin test following partial neutralization with specific soluble blood-group substance 6 was $1: 1,024$ for one lot and $1: 256$ for the other.

\section{Discussion}

The patient reported in this study developed progressive anemia following the transfusion of large volumes of commercial concentrated Factor VIII, having a volume of almost $3,100 \mathrm{ml}$, before detec- tion of the cause of the anemia. During nine days following amputation he received $1,710 \mathrm{ml}$ of the Factor VIII concentrate and this material contained potent anti-A activity. Since there was no overt blood loss, and since the only other product which the patient received was $\mathrm{ABO}$ and $\mathrm{Rh}$-compatible blood, we must conclude that the progressive anemia was due to sensitization of the patient's red blood cells by anti-A contained in the Factor VIII concentrate.

The 43,000 units of the Factor VIII product infused before the amputation did not produce hemolysis. However, this was administered over 40 days. In contrast, the postoperative infusion of 57,000 units was given in nine days, averaging almost $200 \mathrm{ml}$ per day. In retrospect, the low hematocrit noted on the second day after amputation probably was the initial hemolytic manifestation. Unfortunately, a direct antiglobulin test was not performed until the ninth postoperative day, after considerably more concentrate had been administered. By that time anti-A was detectable in the patient's serum.

Mollison has summarized the main features observed in type A patients transfused with plasma containing relatively potent anti-A. ${ }^{3}$ Clinical signs of erythrocyte destruction in adults, such as hemoglobinuria and jaundice occur only in severe cases, and were absent in our patient. However, he manifested mildly increased serum hemoglobin and decreased haptoglobinbinding capacity. Similarly, there was microspherocytosis and increased osmotic fragility. Splenomegaly was not detected.

Progressive anemia in a patient with hemophilia whose $\mathrm{ABO}$ type is $\mathrm{A}, \mathrm{B}$, or AB should suggest this situation. Spontaneous agglutination of blood samples drawn from the patient and microspherocytosis are both strongly suggestive of this condition. Another consistent finding is a positive direct antiglobulin determination, 
indicating IgG coating of red blood cells. This test should be obtained as early as possible in the evaluation of such patients so that therapy may be modified to avoid the offending antibody.

The possibility of this condition must be considered when the clinical and laboratory studies do not support a diagnosis of active bleeding in the hemophilic patient who develops progressive anemia while under antihemophilic therapy. Failure of recognition might even occasion an increase in the offending antihemophilic therapy. When transfusion became necessary in this patient, type $O$ blood in the form of packed cells was administered, and Factor VIII cryoprecipitate prepared from type AB donors was used for corrective therapy.

Although this complication has been recognized in patients receiving large volumes of pooled plasma, it is also obvious that it should be considered in patients other than those of type $O$, who receive large amounts of the pooled Factor VIII concentrates. Such concentrates are prepared from plasma pools, and may contain potent $\mathrm{ABO}$ antibodies.

\section{References}

1. Darnborough, J.: The anti-A antibody content of pooled plasma. J. Clin. Path. 16: 74, 1963.

2. Marder, V. J., and N. R. Shulman: Major surgery in classic hemophilia using Fraction I. Amer. J. Med. 41: 56, 1966.

3. Mollison, P. L.: Blood Transfusion in Clinical Medicine, 4th edition. Philadelphia, F. A. Davis Co., 1967.

4. Oberman, H. A., B. A. Barnes, and P. L. Ginther: Erythrocyte sensitization and anemia due to isoantibodies in lyophilized pooled plasma. JAMA 198: 323, 1966.

5. Rutzky, J., F. Cohen, and W. W. Zuelzer: Anti- $A$ agglutinins in pooled plasma as a cause of hemolytic ancmia. Blood 11: 403, 1956.

6. Technical Methods of the American Association of Blood Banks, fourth edition. Chicago, American Association of Blood Banks, 1966.

7. Topley, E., J. P. Bull, W. Maycock, A. E. Mourant, and D. Parkins: The relation of the isoagglutinins in pooled plasma to the hemolytic anemia of burns. J. Clin. Path. 16: $79,1968$.

L.ouis A. Rosati, M.D., Resident III, Department of Pathology.

Barbara Barnes, M.T. (ASCP) BB, Instructor in Medical Technology, Department of Pathology.

Harold A. Oberman, M.D., Professor, Department of Pathology; Medical Director, Blood Bank, University of Michigan Medical Center, 1395 East Catherine Street, Ann Arbor, Michigan 48104.

John A. Penner, M.D., Associate Professor, Department of Internal Medicine, Simpson Memorial Institute. 